\title{
Optimal Time Scheduling Scheme for Wireless Powered Ambient Backscatter Communications in IoT Networks
}

\author{
Xiaolan Liu, Yue Gao, Fengye $\mathrm{Hu}$
}

\begin{abstract}
In this paper, we investigate optimal schemes to manage time scheduling of multiple modules including spectrum sensing, radio frequency (RF) energy harvesting (RFH) and ambient backscatter communication (ABCom) by maximizing data transmission rate in Internet of Things (IoT) networks. We first detect ambient RF signals with high signal power as the RF resource of $\mathrm{RFH}$ and $\mathrm{ABCom}$ by using spectrum sensing with energy detection techniques. Specifically, compressive sensing is adopted to detect the wideband RF signals with improving spectrum sensing efficiency at the same time. We formulate a joint optimization problem to manage time scheduling parameter and power allocation ratio. In addition, we propose to find the threshold of spectrum sensing for ABCom communications by analyzing the outage probability of backscatter communications. Numerical results demonstrate that the optimal schemes using spectrum sensing are achieved with better transmission rates. The designed time scheduling scheme with compressive sensing is confirmed to be more efficient, and the superiorities become more obvious with the increase of network operation time. Moreover, the optimal scheduling parameters and power allocation ratios are obtained. Simulations illustrate that the threshold of spectrum sensing for backscatter communications is obtained by analyzing the outage probability of backscatter communications.
\end{abstract}

Index Terms-Ambient Backscatter Communication (ABCom), Internet of Things (IoT), Radio Frequency energy Harvesting (RFH), Compressive Spectrum Sensing.

\section{INTRODUCTION}

$\mathbf{T}$ HE internet of things (IoT) is an intelligent network of different kinds of networks, which can connect various devices, smart sensors and actuators to the internet and enable information exchange and sharing among all IoT nodes [1]. It connects a large number of nodes which are distributed in large areas within a complicated and heterogeneous environment to provide different applications, such as smart home [1] and smart city (air quality index monitoring [2]). Therefore, energy supply for such a large number of IoT nodes becomes a critical challenge, which indicating that these nodes have to achieve energy usage in a self-sustainable way. Energy harvesting is emerging as a promising way to provide IoT nodes with continuous energy. Many research studies have confirmed that energy harvesting is a useful way to solve the problem of energylimited batteries in wireless networks [3], especially wireless energy harvesting [4] which uses ambient radio frequency (RF)

X. Liu and Y. Gao are with the Department of Electronic Engineering and Computer Science, Queen Mary University of London, London E1 4NS, UK (email: \{xiaolan.liu, yue.gao\}@qmul.ac.uk).

F. Hu is now with JiLin University, 130012, Changchun, China (email: hufy@jlu.edu.cn). signals to achieve power supplying in IoT networks [5]. On the other hand, low power communication technologies have drawn some attention because they can extend the battery life of nodes by consuming less energy. For instance, lowpower wide-area networks (LPWANs) which include LoRa, Sigfox and narrow band (NB)-IoT are regarded as promising communication technologies in IoT networks [6]-[8]. Specifically, ambient backscatter communication (ABCom), which is achieved by modulating ambient RF signals, is becoming popular due to its characteristic of low power consumption. ABCom doesn't contain energy-consumed circuits since it doesn't need the conventional transmitter circuit [9].

RF energy harvesting (RFH) and ABCom have drawn much attention since they consider readily available RF signals, such as signals from television (TV)/radio broadcasts, mobile base stations and handheld radios [10] as available RF signal resources. The ambient RF resources can be classified into static and dynamic ambient RF resources. TV signals are static RF resources since their power is relatively stable over time. They are promising energy resources with their high transmission power (up to 10kW) [11]. In [12], the researchers designed a novel broadband Yagi-Uda antenna to harvest ambient RF power from DTV (Digital TV) broadcasting signals. A RFH wireless sensor network prototype was designed by harvesting signals from TV tower in [13]. Although static RF resources can provide predictable RF energy, there could be long-term and short-term fluctuations due to service schedule [14]. Dynamic ambient RF resources (e.g., WiFi access point) are produced by RF transmitters that work periodically or change transmit power over time. Therefore, making use of ambient RF sources has to search for available opportunities intelligently in a certain frequency range.

We first introduce the concept of spectrum sensing in this paper to detect the ambient RF resources, e.g., TV signals, by using energy detection techniques. Spectrum sensing was first proposed to detect the signals sent by primary users in a licensed spectrum in cognitive networks [15], which provides more reliable and real-time results for spectrum occupancy. It is categorized into two types according to the bandwidth of the spectrum interest: narrowband and wideband spectrum sensing. The narrowband spectrum sensing algorithm contains energy detection, matched filtering and cyclostationary feature detection [16]. Energy detection is most commonly researched due to its easy implementation and low computational complexity [17]. But for exploiting the wideband spectrum, such as ultra high frequency (UHF) TV band, wideband spectrum 
sensing should be employed since narrowband spectrum sensing cannot be directly used due to its single binary decision making [18]. Alternatively, compressive sensing (CS) theory, first proposed to be applied to wideband spectrum sensing by Tian and Giannakis [19], could be used. Furthermore, a cognitive radio enabled TD-LTE test-bed has been proposed in [20], authors use compressive sensing to detect TV white space to achieve dynamic spectrum management. [21] proposed a blind joint sub-Nyquist sensing scheme to jointly sample the spectrum by utilizing the surround IoT devices in IoT networks based on CS theory. CS uses a small number of measurements to reconstruct the received signals, and is popular due to low cost and high efficiency. To investigate channel energy statistics, a learning algorithm is proposed to explore the assumptive statistical model of channel energy for compressive spectrum sensing [22].

$\mathrm{ABCom}$ is emerging as a potential way to transmit information by reflecting an incident RF wave due to its characteristics of low power (tens of $\mathrm{uW}$ ) and low complexity (without energy hungry circuits) [23]. Furthermore, ambient backscatter communication is used more to communicate with wireless devices nearby since it uses legacies of RF signals (TV, FM and WiFi signals) [24]. Leveraging the ambient RF signals contributes to a self-sustainable IoT network, that is, one which can use the RF signals to power the device and transmit information by using the backscatter technique simultaneously [25]. A communication system that enables two devices to communicate by leveraging existing TV signals was designed in reference [26]. A general framework for evaluating the ultimate achievable rates of a point-to-point backscatter communication network was studied in [27].

However, none of the existing work considers the dynamics of ambient RF signals which are important for performing energy harvesting and ambient backscatter communications. In this paper, we detect ambient RF sources with high power by using spectrum sensing techniques, so that energy harvesting and ABCom can be performed at the IoT node with the filtered $\mathrm{RF}$ resources. This makes the random energy arriving process become a deterministic process, and helps to find the best incident RF signal for ABCom. The major contributions of this paper are:

1) We detect the transmitted signals instead of vacant channels by using compressive spectrum sensing techniques.

2) We propose time scheduling schemes for multiple modules of IoT node by formulating the transmission rate maximization problem of $\mathrm{ABCom}$ process.

3) We analyze the performance of detection threshold of spectrum sensing for $\mathrm{ABCom}$ by analyzing the outage probability of ABCom with considering the channel suffering from path loss, shadowing and fading.

4) The optimal values of time scheduling parameters and power allocation ratios, and the maximum data transmission rates are obtained. The better transmission rate performance by detecting the RF signals with compressive spectrum sensing techniques is verified.

This paper is organized as follows. The network model is presented in section II. The optimal time scheduling schemes

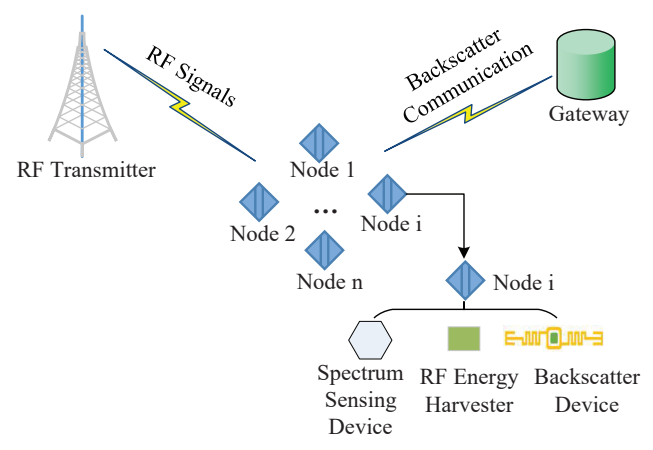

Fig. 1. The network model.

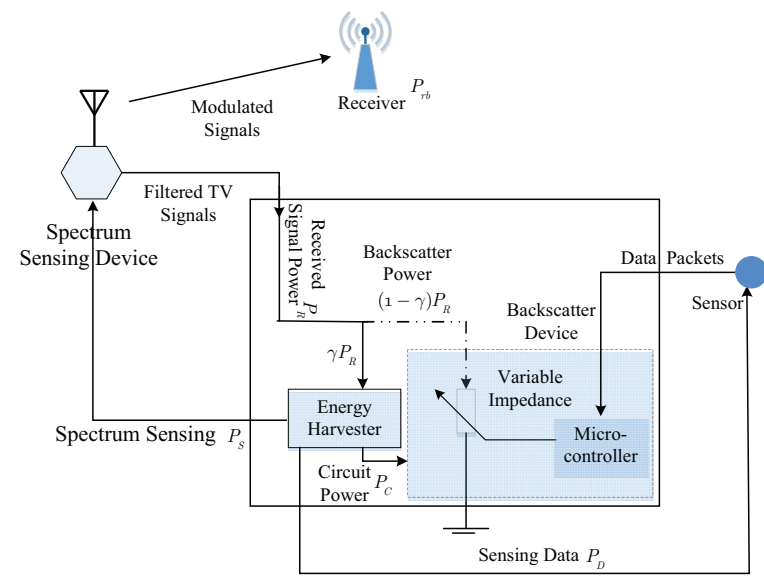

Fig. 2. The block diagram of different modules at the IoT node.

are proposed in section III. The detection threshold of spectrum sensing for ABCom is analyzed in section IV. Numerical results corresponding to our analysis are discussed in section V. Section VI draws the conclusions and future work.

\section{SySTEM MOdEL}

As shown in Fig. 1, we consider an IoT network, which contains a lot of IoT nodes which are powered by ambient RF sources. All the IoT sensor nodes transmit data to the gateway by performing ambient backscatter communications using ambient RF resources. In this paper, multiple modules are considered to be deployed to each IoT node, which helps them achieve energy self-sustainable and make the best use of the received ambient RF sources. The modules include RF energy harvester to harvest RF signals, spectrum sensing to detect ambient RF signals and $\mathrm{ABCom}$ to transmit data to the gateway. Certainly, backscatter receivers are equipped at the end of the gateway to receive the data from all the IoT nodes.

To enable the multiple modules work efficiently at IoT node, a time scheduling scheme is required to ensure the work process of each module. The work process of all the modules at an IoT node is proposed as shown in Fig. 2. The detailed process is formulated as: 1) the spectrum sensing module detects the ambient RF signals with high signal power; 2) the detected RF signals are then used to perform RF energy harvesting or ambient backscatter communications; 3) the energy harvester harvests the RF signals and then 
converts them into direct current (DC); 4) the backscatter device modulates data packets by using the detected high power RF signals, which is achieved by adjusting the variable impedance using the micro-controller. The energy harvested by the RF energy harvester is used to power all the other modules, and we assume that the consumption energy by the $\mathrm{RF}$ energy harvester is negligible. Thus, the energy causal condition should be satisfied, and it can be expressed as:

$$
E_{H} \geq E_{S}+E_{B}+E_{D}
$$

where $E_{H}, E_{S}, E_{B}$ and $E_{D}$ indicate the harvested energy, the energy consumed by the spectrum sensing module, the consumption energy of $\mathrm{ABCom}$ module and the energy consumed by the sensing module, respectively. Assuming all the modules work in the same time block, then how to manage the time scheduling of different modules is a challenging problem. So in this paper, the optimal time scheduling scheme is designed to solve this problem with IoT nodes achieving energy selfsustainable.

\section{Proposed Optimal time Scheduling Scheme}

In this section, the optimal scheme is designed to manage the time scheduling of different modules for each IoT node. We adopt a harvested-then-forward strategy to harvest energy firstly, and then use the harvested energy to transmit data. For detecting single frequency signals, the detected RF signals $(100 \%)$ only can be either used for RFH or modulated $(100 \%)$ by the ABCom module. However, ambient RF sources are generally wideband signals, to detect all the ambient RF signals transmitted in the wideband spectrum, compressive sensing technique is used. As a result, we can detect multiple signals from wideband frequency, and then the detected signals are used to perform RF energy harvesting (partial signals) and $\mathrm{ABCom}$ (the rest signals) simultaneously.

In [13], the TV signals transmitted in the occupied primary channels can be harvested by the RF energy harvester, which is one of the important RF energy resources. In this paper, we consider TV signals as ambient RF resources, with frequency ranging from 470 to $790 \mathrm{MHz}$. The energy detection technique, which is a classic method to detect the occupancy of primary channels by comparing the signal power of the received signals at the second user to a pre-defined power threshold. The detection threshold is set as the minimum value that can be harvested successfully by the RF energy harvester, while for ABCom, the detection threshold is set as a larger value to reduce the outage probability of data transmission. Furthermore, we analyze the value range of the pre-defined detection threshold of spectrum sensing for $\mathrm{ABCom}$ by analyzing the outage probability of ABCom.

Assuming the TV signal received at the IoT node is $y(t)$, which is presented as

$$
y(t)=x(t) h+w_{t},
$$

where $x(t)$ is the received TV signal at the IoT node, $h$ is the channel gain and $w_{t}$ is the noise. Then the power of the

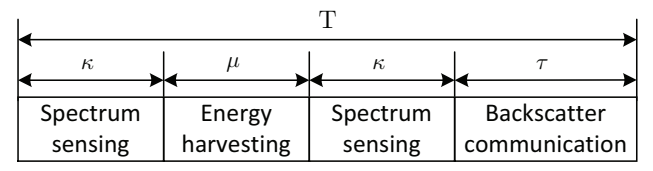

Fig. 3. The time scheduling structure.

received signal is calculated as

$$
P_{R}=\frac{1}{N_{s}} \sum_{n=1}^{N_{s}}|y[n]|^{2},
$$

where $N_{s}$ is the number of samples, $n$ is the index of discrete samples.

\section{A. Optimal Time Scheduling Scheme}

Fig. 3 shows the proposed time scheduling structure of multiple modules for each IoT node, where only one single frequency signal is detected. $T$ is the period of the time block, $\tau$ is the duration of ABCom, while $\mu$ is the duration of RF energy harvesting. The time duration of spectrum sensing is denoted by $\kappa$. Since spectrum sensing is used to detect single frequency signals, it has to detect TV signals every time before RF energy harvesting or ABCom.

Furthermore, each part of the energy causal condition in formula (1) is given respectively. The harvested energy by the $\mathrm{RF}$ energy harvester is given as

$$
E_{H}^{n}=\mu T P_{H}^{n}=\mu T \eta P_{R}^{n},
$$

where $\eta$ is the energy harvesting efficiency and $P_{R}^{n}$ is the received signal power at the IoT node.

The consumption energy of the spectrum sensing module is presented as

$$
E_{\mathrm{S}}^{n}=e_{s} N_{s} N_{n},
$$

where $N_{n}$ is the number of signals detected during spectrum sensing, and $e_{s}$ denotes the power consumed by each sample.

Assuming the pre-defined detection threshold for energy harvesting is $\lambda_{h}$, the RF energy harvester starts to harvest energy only when the detected signal power $P_{R}^{n} \geq \lambda_{h}$. And if the pre-defined detection threshold for ABCom module is assumed as $\lambda_{b}$, then it performs backscatter communications only when the detected signal power $P_{R}^{n} \geq \lambda_{b}$. Supposing the sampling rate of each signal is the same, denoted by $f_{s}$, so the total number of signals detected by the spectrum sensing module during $2 \kappa T$ is $N_{n}=2 \kappa T f_{s} / N_{s}$. Thus, the energy consumed by spectrum sensing during one time block $T$ is changed as

$$
E_{\mathrm{S}}^{n}=e_{s} 2 \kappa T f_{s} .
$$

The consumption energy of the ABCom module is mainly used to power the circuit of modulating data packets, so it is expressed as

$$
E_{B}^{n}=\tau T P_{C}
$$

where $P_{C}$ denotes the circuit power of the ABCom module. For simplicity, the consumption energy of sensing data $E_{D}$ is set as a constant. 
To design the optimal time scheduling scheme, we formulate the transmission rate maximization problem, where our objective function is the transmission rate of $\mathrm{ABCom}$, which is presented as

$$
R_{n}(\tau)=\tau T B_{w} \log _{2}\left(1+\frac{P_{U}^{n}}{W_{n}}\right),
$$

where $P_{U}^{n}$ is the received signal power of the backscatter receiver at the data server, $W_{n}$ is the noise power. Since modulated signals by the backscatter module will experience path loss before arriving at the backscatter receiver, $P_{U}^{n}$ is written as $P_{U}^{n}=\beta P_{R}^{n} L\left(d_{1}\right) . P_{R}^{n}$ is the received signal power after detecting by the spectrum sensing module, $\beta$ is the reflection coefficient of backscatter communications. In addition, it also suffers from path loss from the TV tower to the IoT node, which is denoted as $P_{R}^{n}=P_{o} L\left(d_{2}\right), P_{o}$ is the power of TV signal at the transmitter. $L(d)=B d^{-\varsigma}$ is the power-law path loss exponent. The path loss function depends on the distance $d$, a frequency dependent constant $B$ and an environment dependent path-loss exponent $\varsigma \geq 2$.

Then the optimization problem is formulated as:

$$
\begin{array}{ccc}
O P 1 \max _{\tau} & R_{n}(\tau), \\
\text { s.t. } & 0<\tau, \kappa, \mu<1, \\
& \tau+2 \kappa+\mu=1, \\
& E_{H}^{n} \geq E_{S}^{n}+E_{B}^{n}+E_{D},
\end{array}
$$

where $E_{D}=P_{D} T, P_{D}$ is the power of the data sensing module. By substituting formulas (4), (6) and (7) into the optimization problem OP1, we can get the OP2 as follows:

$$
\begin{aligned}
O P 2 \max _{\tau} \quad & R_{n}(\tau), \\
\text { s.t. } \quad & 0<\tau, \kappa, \mu<1, \\
& \tau=1-2 \kappa-\mu, \\
\tau \leq & \frac{\mu \eta P_{R}^{n}-e_{s} 2 \kappa f_{s}-P_{D}}{P_{C}} .
\end{aligned}
$$

In the optimization problem OP2, we can see that $R_{n}(\tau)$ is a monotonically increasing function with $\tau$. In the ideal case, $\tau$ is as close as possible to 1 , that is, $\kappa$ and $\mu$ are close to 0 . However, the time of RF energy harvesting is not negligible since it only harvests a small amount of power. Then if we let $\kappa=0$, then $\mu=1-\tau$. Therefore, we can get the maximum transmission rate $R_{n}^{*}(\tau)$ when $\tau=\frac{\eta P_{R}^{n}-P_{D}}{\eta P_{R}^{n}+P_{C}}$. In practice, spectrum sensing process should be considered, that is, $\kappa \neq 0$. Therefore, to solve this optimization problem with multiple variables, a grid search algorithm is used as shown in Algorithm 1.

The complexity of our proposed algorithm mainly comes from the search grid space, let $N_{\kappa}$ and $N_{\mu}$ present the grid space of $\kappa$ and $\mu$, respectively. Therefore, the complexity of our proposed time scheduling scheme is $O\left(N_{\kappa} \times N_{\mu}\right)$.

\section{B. Time Scheduling Scheme with Compressive Sensing}

In this section, compressive sensing is considered to detect wideband signals from the TV spectrum. In this case, we can obtain more than one TV signals from different channels at the same time.

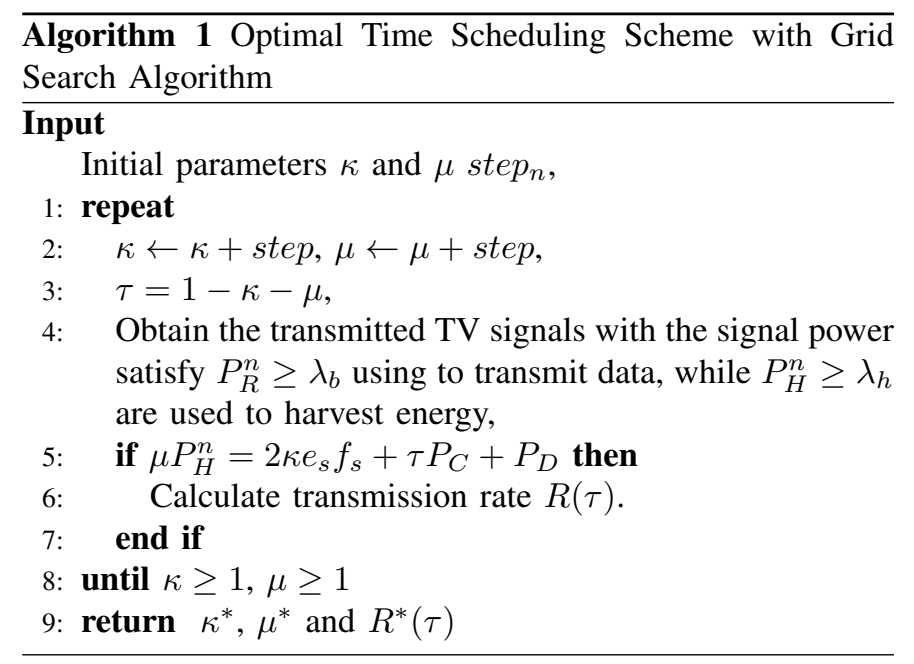

1) Compressive Sensing: Compressive sensing technique is efficiently used to acquire and reconstruct a signal, which requires the signal is sparse in some domain. Then the sparsity of a signal is exploited to recover it from far fewer samples than required by the Shannon-Nyquist sampling theorem through some optimization methods. The TV wideband spectrum in the UK has abundant spectrum resources with each channel bandwidth is $8 \mathrm{MHz}$, and most of the channels are rarely used. We consider this wideband TV signal as sparse signal, which is indicated by $S=\left(s_{1}, s_{2}, \ldots, s_{M}\right)$. Assuming there are $K$ active channels occupied during sensing period, the utilisation ratio is defined as $\xi$, then $K=\xi \cdot M$. Therefore, applying compressive sensing to the TV wideband spectrum sensing, we can obtain the information of TV signals transmitted in the active occupied channels.

Assuming the received signal $y(t)$ shown in formula (2) at the IoT node is wideband signal. Moreover, we assume the received signal is sparse in the frequency domain and it is expressed as $y_{f}=x_{f} h_{f}+w_{f}$, where $y_{f}, x_{f}$ and $w_{f}$ are the discrete Fourier transform (DFT) of $y(t), x(t)$ and $w(t)$. Also, $x_{f}$ is sparse because the spectrum is underutilised. Through applying compressive sensing technique at the IoT node, the collected compressed measurements can be presented as

$$
c=\Phi F^{-1} y_{f}=\Phi F^{-1}\left(x_{f}+w_{f}\right),
$$

where the measurement matrix $\Phi \in C^{K \times M}$ is used to collect the compressed measurements $c \in C^{K \times 1}$, with the compressive ratio $\xi=\frac{K}{M}$. And $F^{-1}$ is inverse DFT matrix which is used as the sparsifying matrix. The sub-Nyquist sampling technology is adopted when the compressive sensing is implemented at the IoT node, the sampling rates are reduced then. To detect the occupancy of the active channels, the received signal should be recovered from the compressed measurements, it can be achieved by solving the following $l_{1}$ norm minimization problem:

$$
\hat{x}_{f}=\arg \min \left\|\hat{x}_{f}\right\| \text {, s.t. }\left\|\Phi F^{-1} \hat{x}_{f}-c\right\|_{2}^{2} \leq \varepsilon,
$$

where $\varepsilon$ is the noise tolerance. Further, energy detection method is performed at the IoT node to determine the occupancy of the active channels, the pre-defined threshold is 


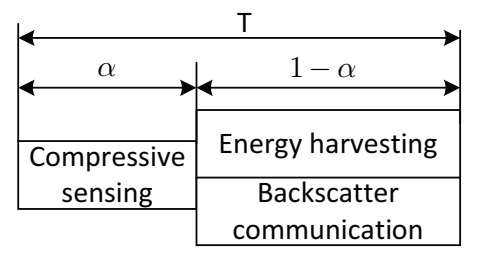

Fig. 4. The time scheduling structure with compressive sensing.

given by

$$
\lambda=\sigma_{d}^{2} *\left(1+\frac{Q^{-1}\left(P_{f}\right)}{\sqrt{M / 2}}\right),
$$

where $P_{f}$ is the probability of false alarm. So if the power density of each considered channel satisfies $P_{\hat{x}_{f}}>\lambda$, then there is TV signal transmitted in this channel, otherwise the channel is vacant.

2) Time Scheduling Scheme: The received power at the IoT node is split into two parts, one is used to backscatter information, another part is harvested by the RF energy harvester, as shown in Fig. 2. This means that energy harvesting and backscatter communication are performed simultaneously after compressive sensing. And the predefined detection threshold of energy detection is set as the bigger value of the two processes as defined in the previous time scheduling scheme. In this case, the time scheduling structure is designed as shown in Fig. 4. $T$ is the period of the time block. $\alpha$ denotes the time duration of compressive sensing, while $1-\alpha$ indicates the time duration of energy harvesting and the ABCom.

Similarly, the harvested energy is used to power the compressive sensing module, the ABCom module and the sensor module. Here, only one part of the received power, which is indicated by power allocation ratio $\iota$, is used for energy harvesting, then the harvested energy is formulated as

$$
E_{H}^{w}=(1-\alpha) T \eta \iota P_{\hat{x}_{f}},
$$

where $P_{\hat{x}_{f}}$ is the power of the signals recovered from compressive sensing.

The consumption energy of the compressive sensing module is expressed as

$$
E_{S}^{w}=e_{s} f_{s} M \alpha T,
$$

where $e_{s}$ is the consumption energy of each sample, $f_{s}$ is the sample rate of each signal.

The energy consumed by the ABCom module is presented as

$$
E_{B}^{w}=(1-\alpha) T P_{C}
$$

In this case, the transmission rate of the backscatter communications is relevant to time scheduling of each module and the power allocation ratio between energy harvesting and backscatter communications, then it is represented as

$$
R_{w}(\alpha, \iota)=(1-\alpha) T B \log _{2}\left(1+\frac{\beta(1-\iota) P_{\hat{x}_{f}} L\left(d_{2}\right)}{W_{w}}\right),
$$

where $(1-\iota) P_{R}^{w}$ indicates the part of received power used for backscatter communications. $L\left(d_{2}\right)$ is the power-law path loss between the IoT node and the backscatter receiver.

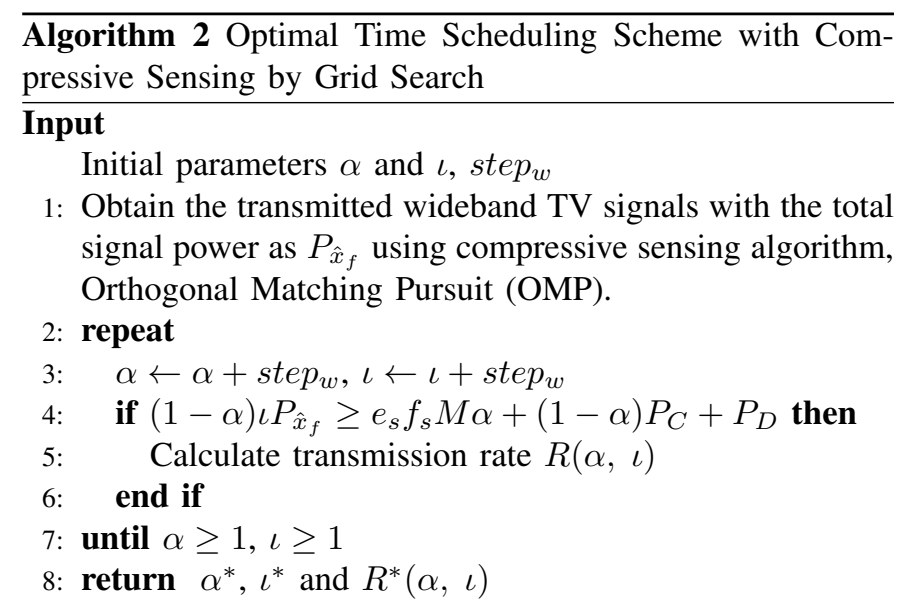

Therefore, we formulate a joint optimization problem to optimize $\alpha$ and $\iota$, which is given as

$$
\begin{aligned}
& O P 3 \max _{\alpha, \iota} R_{w}(\alpha, \iota), \\
& \text { s.t. } \quad 0<\alpha, \iota<1, \\
& E_{H}^{w} \geq E_{S}^{w}+E_{B}^{w}+E_{D}^{w} .
\end{aligned}
$$

Similarly, where $E_{D}$ is the energy consumed by the sensor sensing data. Substituting formulas (14), (15) and (16) into this optimization problem, it is reformulated as

$$
\begin{aligned}
& O P 4 \max _{\alpha, \iota} \quad R_{w}(\alpha, \iota), \\
& \text { s.t. } \quad 0<\alpha, \iota<1, \\
&(1-\alpha) \eta \iota P_{R}^{w} \geq e_{s} f_{s} M \alpha+(1-\alpha) P_{C}+P_{D} .
\end{aligned}
$$

Likewise, the compressive sensing takes up as less time as possible in the ideal case, that is, $\alpha=0$. Then the joint optimization problem is simplified as the transmission rate only varies with the power allocation ratio $\iota$. In addition, the transmission rate is monotonically decreasing with $\iota$, so the optimal transmission rate $R_{w}^{*}(\iota)$ is obtained with $\iota=\frac{P_{C}+P_{D}}{\eta P_{x_{f}}}$. However, in practice, the time scheduled for compressive sensing cannot be negligible, then we use grid search to solve this optimization problem. The solution for this optimization problem using grid search algorithm is shown in Algorithm 2.

The complexity of the compressive sensing is determined by the number of samples $M$ to be recovered to present the spectrum of interest, and it is from the signal recovery process by solving (12) and denoted by $O\left(M^{3}\right)$. The complexity of our proposed algorithm mainly comes from the search grid space, let $N_{\alpha}$ and $N_{\iota}$ present the grid space of $\alpha$ and $\iota$, respectively. Therefore, the complexity of our proposed time scheduling scheme is $O\left(M^{3}+N_{\alpha} \times N_{\iota}\right)$.

\section{Optimal Dection Threshold For ABCom}

In Section III, we have designed an optimal time scheduling schemes of multiple modules at an IoT node. In the optimal scheme, spectrum sensing technique is first used to detect the transmitted TV signals instead of the vacant frequencies. The signals are detected by changing the detection threshold of energy detection technique, which then are used to perform energy harvesting and ABCom. The pre-defined detection threshold for energy harvesting is set as the minimum value 
that can be harvested by the RF energy harvester. But for ABCom, the pre-defined detection threshold is decided by the interferences of other IoT nodes and the channel condition between backscatter device and the receiver. Therefore, we discuss the upper and lower bound of the pre-defined detection threshold of spectrum sensing for ABCom in this section.

As shown in Fig. 1, the IoT network contains many IoT nodes distributed around the gateway (which is considered as the local data server). If these nodes transmit data with high transmission power, they will cause interferences to each other. This means the transmit power of each IoT node should be no more than the maximum power. Thus, the upper bound of the pre-defined detection threshold is existed. It is obtained by maximizing the transmission rate of ABCom. For simplicity, we assume the transmit power of each IoT node is the same, then the transmission rate is presented as

$$
\begin{aligned}
R_{b} & =B \log _{2}\left(1+\frac{P_{l} h_{b}}{W_{0}+\sum_{j=1, j \neq l}^{J} P_{j} g_{j}}\right) \\
& =B \log _{2}\left(1+\frac{P_{l} h_{b}}{W_{0}+(K-1) P_{l} \sum_{j=1, j \neq l}^{J} g_{j}}\right),
\end{aligned}
$$

where $W_{0}$ is the noise power of the receiver, and $P_{l}=P_{j}$ is the transmission power of IoT node. $h_{b}$ is the channel gain from backscatter module to the receiver, $g_{j}$ is the channel gain from the rest IoT nodes to the IoT node $l$. And as shown in formula (20), the transmission rate $R_{b}$ is monotonically increasing with the transmit power $P_{l}$. Generally, the upper bound of the transmit power is limited by battery capacity of the IoT node. In this paper, since the backscatter module transmits data by reflecting the transmitted TV signals, the upper bound of transmit power is decided by the maximum power of the TV signals detected by the spectrum sensing module.

Furthermore, we discuss the lower bound of the pre-defined detection threshold which ensures successful data transmission from the backscatter module to the receiver. In reality, the channel between them suffers from large-scale fading (including path loss and shadowing) and small-scale fading (like Rayleigh and Nakagami-m fading). In this paper, we consider all the fading characteristics of the channel to find the predefined detection threshold of spectrum sensing for ABCom, that is, the transmit power of the IoT node is analyzed.

Firstly, we use the simplified path loss model to illustrate the influences of the communication distance on the transmit power, it is expressed as

$$
P_{t}=\frac{P_{R}^{b}}{B d^{-\varsigma}} \geq \frac{P_{t h}}{B d^{-\varsigma}},
$$

where $P_{t h}$ is the threshold of the received power at the receiver. Then we can use formula (21) to model the path loss of the signals before they arrived the receiver. Then it is easy to obtain the minimum transmit power according to the received power since which should be larger than a minimum threshold to make sure the successful data transmission.

Then, we consider finding $P_{t h}$ by analyzing the outage probability of the channel with composite Nakagami-m fading and log-normal shadowing. This is to analyze the effects of shadowing and fading in the channel on the received power. Firstly, the definition of the outage probability is presented as

$$
P_{\text {out }}=\operatorname{P}_{r}\left(\gamma<\gamma_{t h}\right)=\int_{0}^{\gamma_{t h}} p_{\gamma}(\gamma) d_{\gamma},
$$

where $\gamma$ is the instantaneous signal-noise ratio (SNR) and $\gamma_{t h}$ is the minimum SNR that must be satisfied at the receiver. The composite probability density function (PDF) with Nakagami$\mathrm{m}$ fading and log-normal shadowing of SNR is presented as [28]

$$
\begin{aligned}
& p_{\gamma}(\gamma)=\int_{0}^{\infty} \frac{m^{m} \gamma^{m-1}}{\Omega^{m} \Gamma(m)} \exp \left(-\frac{m \gamma}{\Omega}\right) \\
& \quad \times\left\{\frac{10 / \ln 10}{\sqrt{2 \pi \sigma^{2}} \Omega} \exp \left[-\frac{\left(10 \log _{10} \Omega-\mu\right)}{2 \sigma^{2}}\right]\right\} d \Omega, \quad \gamma \geq 0,
\end{aligned}
$$

where $\Omega$ is the average power which is treated as a random variable, and $m$ is the parameter of Nakagami-m fading. $\sigma(d B)$ and $\zeta(d B)$ are the mean and standard deviation of $10 \log _{10} \Omega$, respectively. Then the outage probability is obtained as

$$
\begin{aligned}
& P_{\text {out }}=\int_{0}^{\gamma_{t h}} \int_{0}^{\infty} \frac{m^{m} \gamma^{m-1}}{\Omega^{m} \Gamma(m)} \exp \left(-\frac{m \gamma}{\Omega}\right) \\
& \quad \times\left\{\frac{10 / \ln 10}{\sqrt{2 \pi \sigma^{2}} \Omega} \exp \left[-\frac{\left(10 \log _{10} \Omega-\mu\right)}{2 \sigma^{2}}\right]\right\} d \Omega d \gamma
\end{aligned}
$$

In formula (22), the instantaneous SNR $\gamma$ is presented as

$$
\gamma=\frac{\rho^{2} E_{s}}{N_{0}}=\frac{\rho^{2} P_{R}^{b} T_{s}}{W_{0} / B}=\frac{\rho^{2} P_{R}^{b}}{W_{0}} \quad(a),
$$

the SNR threshold $\gamma_{t h}$ is

$$
\gamma_{t h}=\frac{\rho^{2} P_{t h}}{W_{0}}
$$

where $\rho^{2}$ is the fading power, $E_{s}$ indicates the energy per symbol and $N_{0}(W / H z)$ is the one-sided power spectral density at the receiver. $P_{R}^{b}$ is the received signal power and $W_{0}$ is the noise power at the receiver. $T_{s}$ is the period of the signal and $B_{s}$ is the bandwidth of the noise, and it satisfies $B_{s}=\frac{1}{T_{s}}$, so we can get the final expression in formula (25-a).

By substituting formula (25-a) into (24), we can get the outage probability varying with the received signal power $P_{R}^{b}$ which is the signal power before experiencing fading. Thus, the threshold of this power can be obtained by analyzing the outage probability shown in formula (24), that is, the threshold of $P_{t h}$ in formula (21) is obtained. Finally, to calculate the threshold of the transmission power, we can analyze the formula (21) since the value range of the received power $P_{t h}$ is known now.

\section{NumERicAl RESUlts}

In this section, simulations are performed for optimal time scheduling schemes proposed in Section II. For comparison, the relevant simulation parameters are set as the same, $T=10 \mathrm{~s}$, the consumption energy of the sensor is $P_{D}=$ $-30 \mathrm{dBm}$, and the circuit power of backscatter module is $P_{C}=-40 \mathrm{dBm}$. The consumption energy of each sample is $e_{s}=-33 \mathrm{dBm}$. The path-loss exponent for both communication links is $\varsigma=2$. The sampling rate of each signal is $f_{s}=1000 \mathrm{~Hz}$, and the noise power at the backscatter receiver is given as $W_{n}=W_{m}=W_{0}=-40 \mathrm{dBm}$. Simulations are performed to find the detection threshold of spectrum sensing for $\mathrm{ABCom}$. 


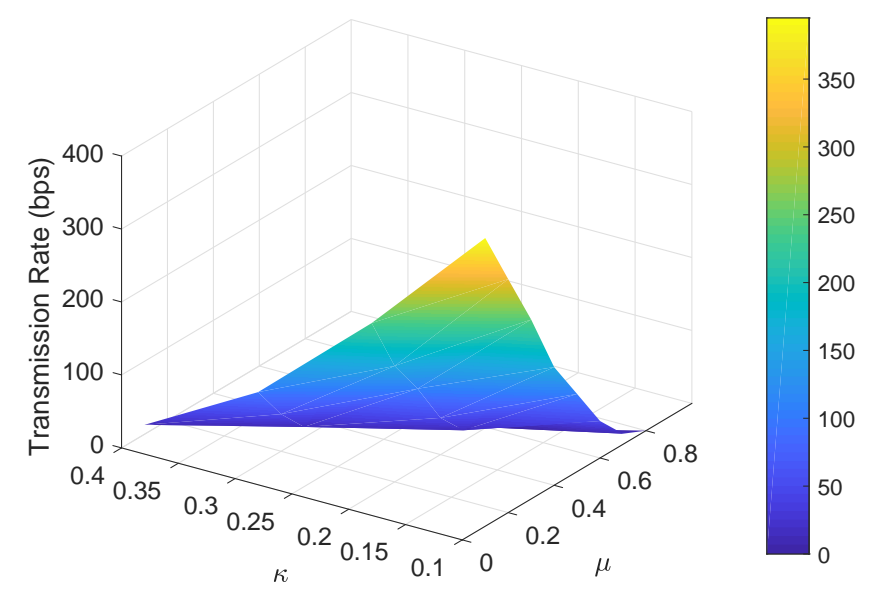

Fig. 5. The transmission rate with spectrum sensing, $\kappa$ is the duration of spectrum sensing, and $\mu$ indicates the duration of energy harvesting.

\section{A. Optimal Scheme with Spectrum Sensing}

In this section, we detect the signals from a single frequency which is set as $562 \mathrm{MHz}$. By solving the optimization problem OP1, the optimal transmission rate of ABCom is obtained with optimal time shceduling parameters, which is shown in Fig. 5. In contrast, Fig. 6 plots the transmission rate of ABCom without adopting the energy detection technique. In this case, it means that the energy harvesting efficiency $\eta$ and the reflection coefficient $\beta$ of $\mathrm{ABCom}$ are smaller than 1, which we set as 0.5 . This is because the received signals that don't satisfy the energy harvesting or ABCom condition will be eliminated. The optimal values of transmission rates and the corresponding optimal time scheduling parameters are given in Table 1(a).

Comparing Fig. 5 to Fig. 6, we can see that the transmission rate is improved. This is because we use a predefined detection threshold to filter the received signals for energy harvesting and $\mathrm{ABCom}$. So in this case, $\eta$ and $\beta$ are equal to 1 , which means all the filtered signals arrived in the energy harvesting module and the backscatter module can be harvested and modulated successfully. We also illustrate the optimal value ranges of time sheduling parameters $\kappa$ and $\mu$ in these two scenarios.

\section{B. Optimal Scheme with Compressive Sensing}

To improve the spectrum sensing efficiency, compressive sensing is used to detect the wideband TV signals. There are 40 channels ranging from 470 to $790 \mathrm{MHz}$, so the maximum number of signals transmitted in the wideband spectrum is 40. But most of the channels are rarely used, so the wideband signal is sparse, and the sparsity is set as $\xi=75 \%$. We use the classical recovery algorithm Orthogonal Matching Pursuit (OMP) to recover the original wideband signal from the sparse measurements.

For comparison, leveraging this wideband signal without compressive sensing is also performed. Similarly, the energy harvesting efficiency $\eta$ and the reflection coefficient $\beta$ are set as 0.5 in this scenario. In this scheme, both the time

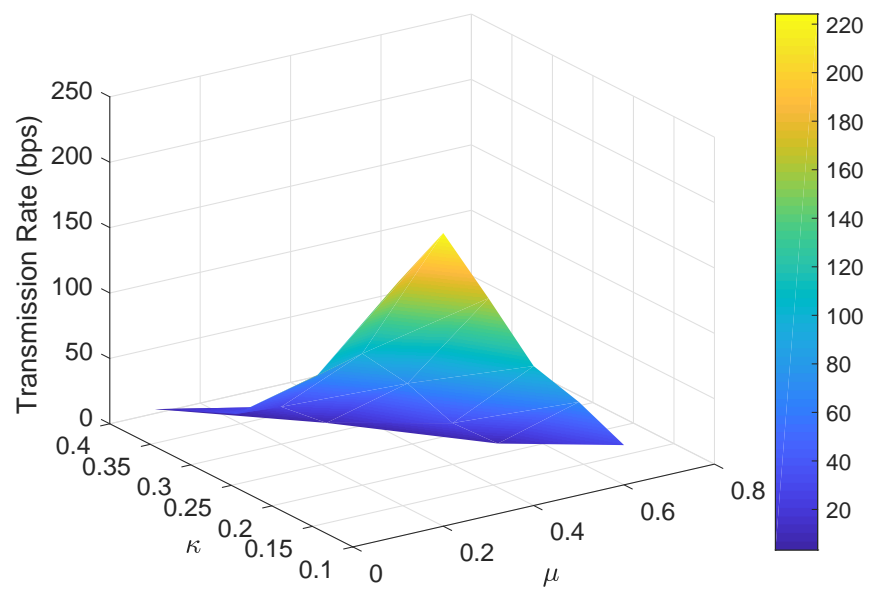

Fig. 6. The transmission rate without spectrum sensing, $\kappa$ is the duration of spectrum sensing, and $\mu$ indicates the duration of energy harvesting.

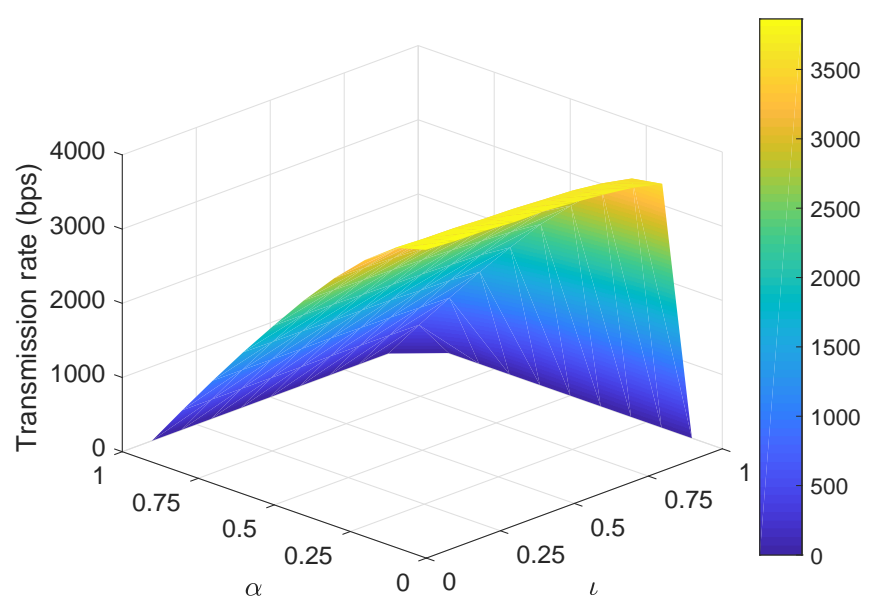

Fig. 7. The transmission rate with compressive sensing, $\alpha$ is the time scheduling parameter, while $\iota$ is the power ratio parameter.

scheduling parameter and the power allocation ratio affect the transmission rate. The obtained optimal values of both parameters and the corresponding transmission rates in both scenarios are given in Table 1(b).

Comparing Fig. 7 to Fig. 8, we can see that the transmission rate with compressive sensing is improved. Likewise, this is

TABLE I

OPTIMAL VALUES OF BOTH SCHEMES

(a) Optimal values with spectrum sensing

\begin{tabular}{|c|c|c|c|c|}
\hline & $R_{n}$ (bps) & $\kappa$ & $\mu$ & $\tau$ \\
\hline with spectrum sensing & 395 & 0.11 & 0.11 & 0.78 \\
\hline without spectrum sensing & 224 & 0.11 & 0.21 & 0.68 \\
\hline
\end{tabular}

(b) Optimal values with compressive sensing

\begin{tabular}{|c|c|c|c|}
\hline & $R_{w}(b p s)$ & $\alpha$ & $\iota$ \\
\hline with compressive sensing & 3864 & 0.11 & 0.11 \\
\hline without compressive sensing & 2694 & 0.21 & 0.11 \\
\hline
\end{tabular}




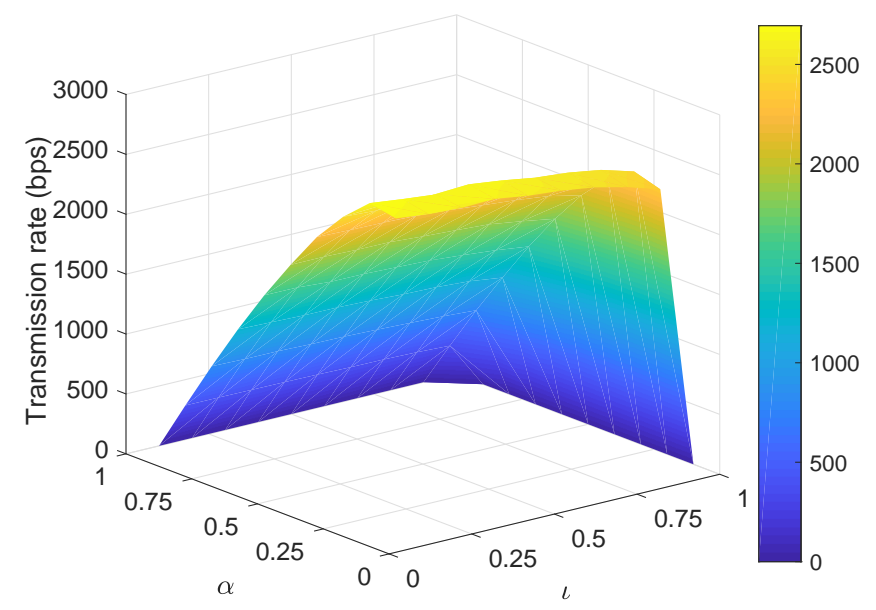

Fig. 8. The transmission rate without compressive sensing, $\alpha$ is the time scheduling parameter, while $\iota$ is the power ratio parameter.

because all the RF signals arrived in the energy harvesting module and the backscatter module are filtered by using the energy detection technique. The performance of the transmission rate is obviously improved with compressive sensing, which is shown in Fig. 5 and Fig. 7. The reason is that the wideband multi-frequency signal can be detected at the same time with compressive sensing, which increases the incident power of energy harvesting and ABCom.

In addition, as shown in Fig. 9, we can easily see that the optimal scheme with spectrum sensing technique has higher transmission rate, and by using compressive sensing, the performance is improved obviously. Fig. 9 also illustrates that the superiority of the scheme using spectrum sensing becomes more obvious with the increase of the network operation time, and the superiority of the time scheduling scheme with compressive sensing has the same trend. Therefore, we can learn that the time scheduling scheme with compressive sensing sacrifices the complexity which is analyzed in Section IV to get better performance of transmission rate than the scheme with spectrum sensing.

\section{Detection Threshold for ABCom}

In this section, simulations are performed to obtain the detection threshold of spectrum sensing module for ABCom. It is calculated by the reverse derive method, firstly, we analyze the outage probability of ABCom suffering composite lognormal shadowing and Nakagami-m fading through formula (24). Then by analyzing the outage probability of ABCom using path loss model through formula (21), we can obtain the detection threshold of spectrum sensing for ABCom. The simulation parameters are set as: the shadowing scenario is set as average shadowing, where $\zeta=-0.115$ and $\sigma=0.161$, and the channel fading amplitude $\rho=0.7$ [29].

As shown in Fig. 10, the outage probability is increasing with the increasing of the SNR threshold $\gamma_{t h}$ at the receiver. According to the definition of the outage probability, it has higher possibility to suffer outage with higher SNR

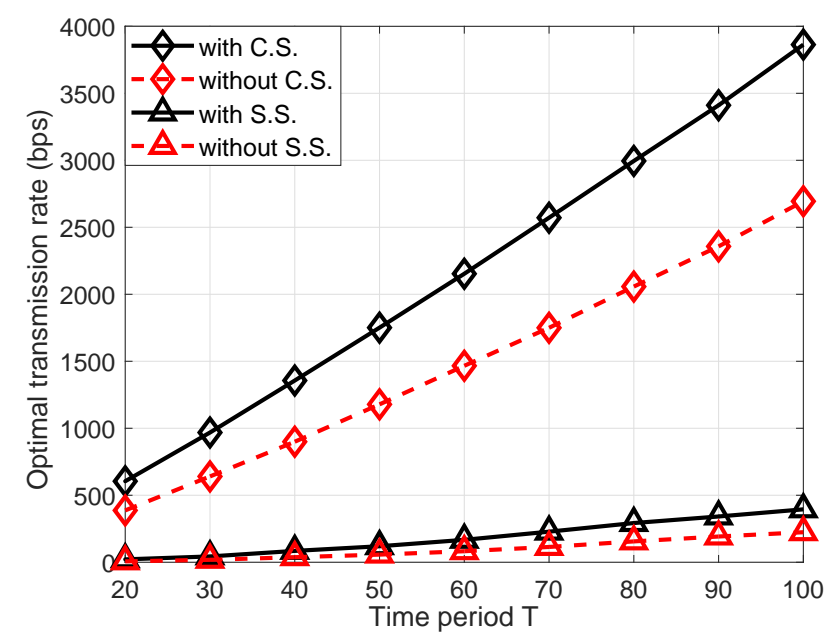

Fig. 9. The transmission rate with increasing network operation time under different time scheduling schemes.

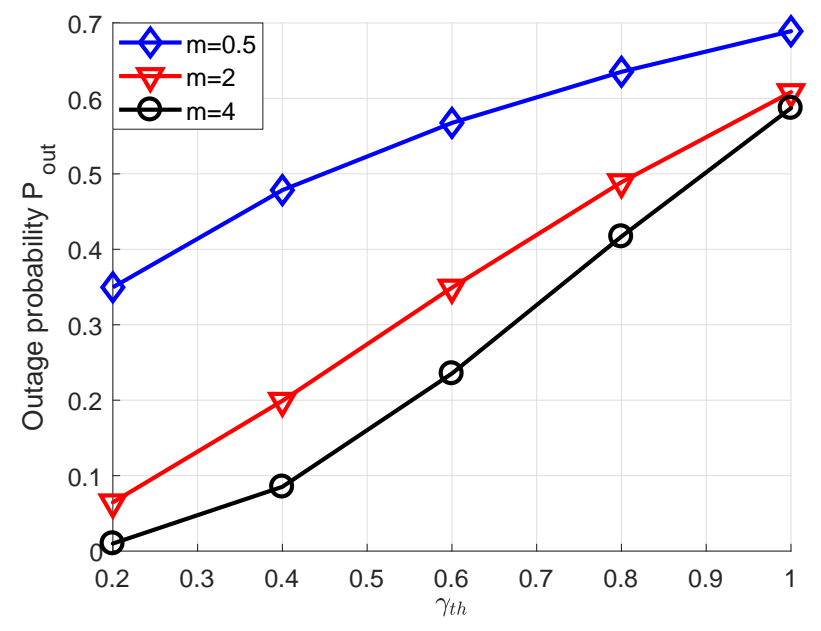

Fig. 10. Outage probability $P_{\text {out }}$ vs SNR threshold $\gamma_{t h}$ with different fading parameters $m$.

requirements. And in formula (25), the SNR threshold $\gamma_{t h}$ and the received power $P_{t h}$ are in linear relation, so the outage probability varying with $P_{t h}$ is easily plotted in Fig.11. It shows that the $P_{\text {out }}$ decreases with the received power $P_{t h}$ increasing under different Nakagami-m fading parameters. And with the increasing of the fading parameter $m$, the $P_{\text {out }}$ is decreased. This is because the Nakagami-m fading converges to a non-fading AWGN channel with $m$ increasing to $+\infty$.

Afterwards, by analyzing the path loss model between the transmission power and the received power shown in formula (21), we obtain the outage probability varying with the transmit power as shown in Fig. 12, that is, the detection threshold of spectrum sensing for ABCom can be determined by controlling the outage probability. And Fig. 13 illustrates the analogous performance of $P_{\text {out }}$ with $P_{t h}$ over different distances between the backscatter module and the receiver. It is obvious that the communication link tends to be interrupted with the increasing of the communication distance. 


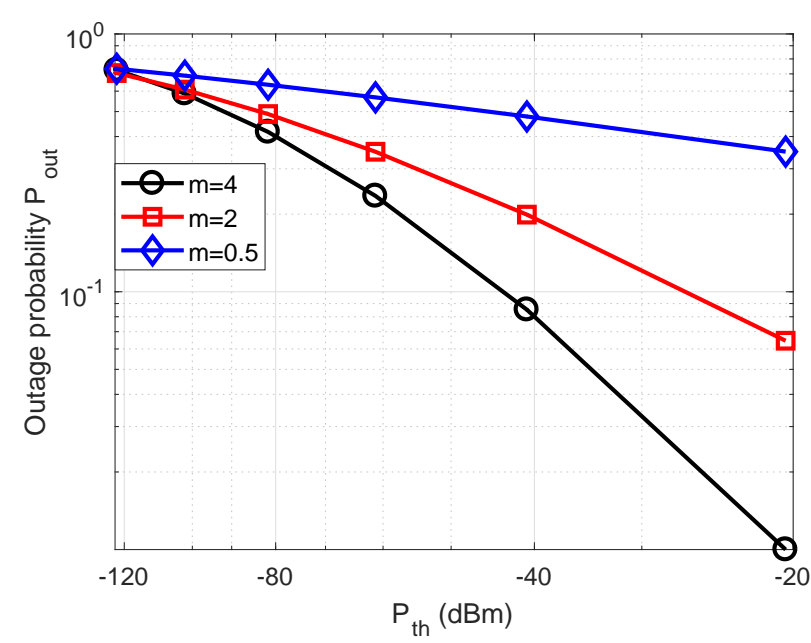

Fig. 11. Outage probability $P_{\text {out }}$ vs received power $P_{t h}$ with different fading parameters $m$.

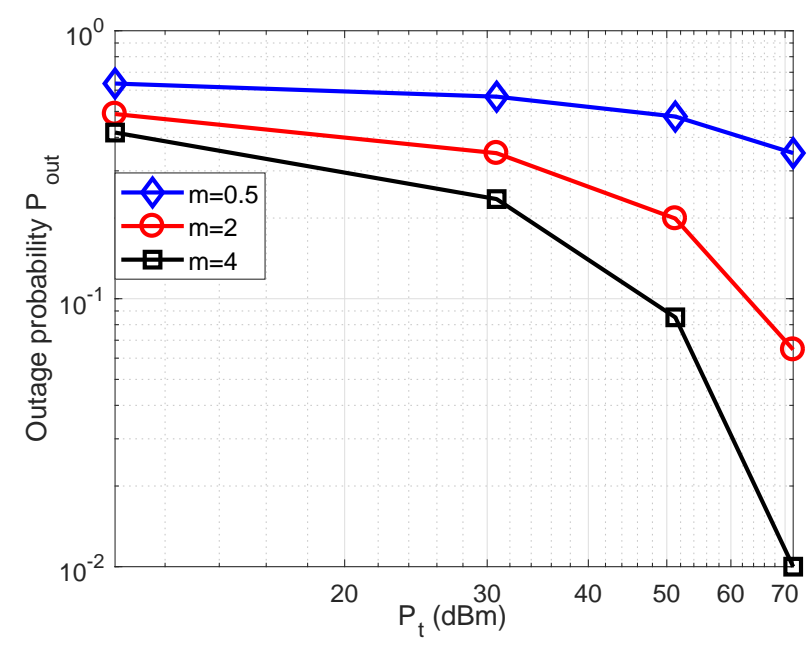

Fig. 12. Outage probability $P_{\text {out }}$ vs transmission power $P_{t}$ with different fading parameters $m$.

\section{CONCLUSION}

In this paper, the optimal time scheduling scheme based on compressive spectrum sensing technique has been proposed to manage time scheduling of spectrum sensing module, the energy harvesting module and the $\mathrm{ABCom}$ module at an IoT node. By maximizing the transmission rate of $\mathrm{ABCom}$, optimal time scheduling parameters and the optimal power allocation ratio have been obtained. Simulations demonstrated that larger transmission rates have been achieved when using spectrum sensing techniques, while compressive sensing has achieved even better performance. The superiorities become more obvious when increasing the network operation time. Finally, we obtained the detection threshold of spectrum sensing for enabling $\mathrm{ABCom}$ by analyzing the outage probability of $\mathrm{ABCom}$.

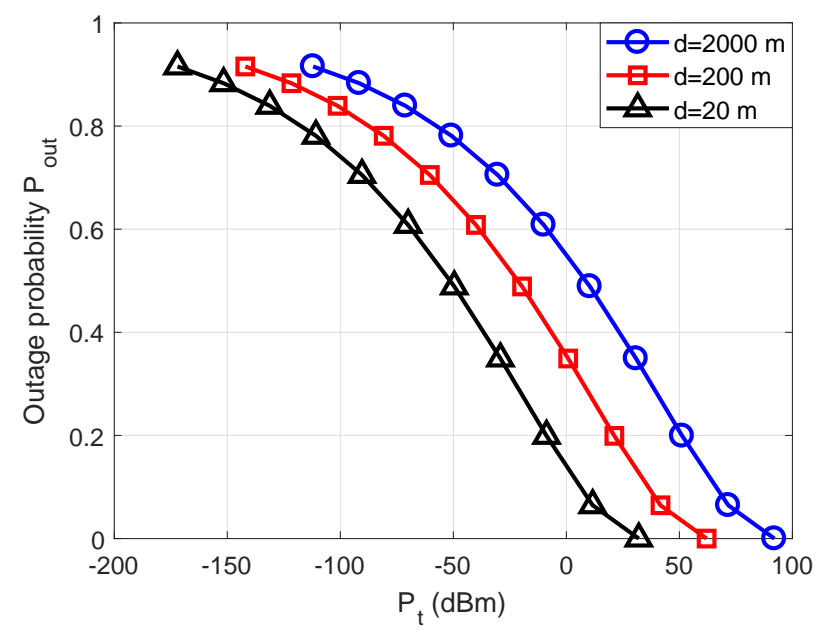

Fig. 13. Outage probability $P_{\text {out }}$ vs transmission power $P_{t}$ with different distances $d$.

\section{REFERENCES}

[1] O. Vermesan and P. Friess, Internet of things: converging technologies for smart environments and integrated ecosystems. River Publishers, 2013.

[2] Y. Yang, Z. Zheng, K. Bian, L. Song, and Z. Han, "Real-time profiling of fine-grained air quality index distribution using uav sensing," IEEE Internet Things J., vol. 5, no. 1, pp. 186-198, Nov. 2018.

[3] S. Kim, R. Vyas, J. Bito, K. Niotaki, A. Collado, A. Georgiadis, and M. M. Tentzeris, "Ambient rf energy-harvesting technologies for selfsustainable standalone wireless sensor platforms," Proc. IEEE, vol. 102, no. 11 , pp. 1649-1666, Oct. 2014.

[4] P. Kamalinejad, C. Mahapatra, Z. Sheng, S. Mirabbasi, V. C. Leung, and Y. L. Guan, "Wireless energy harvesting for the internet of things," IEEE Commun. Mag., vol. 53, no. 6, pp. 102-108, Jun. 2015.

[5] D. Miorandi, S. Sicari, F. De Pellegrini, and I. Chlamtac, "Internet of things: Vision, applications and research challenges," Ad Hoc Networks, vol. 10, no. 7, pp. 1497-1516, Sep. 2012.

[6] M. C. Bor, U. Roedig, T. Voigt, and J. M. Alonso, "Do lora low-power wide-area networks scale?" in Proc. of ACM Intl. Conf. .Modeling, Analysis and Simul. Wireless and Mobile Systems, (MSWiM19), pp. 5967, Nov. 2016.

[7] M. Lauridsen, B. Vejlgaard, I. Z. Kovács, H. Nguyen, and P. Mogensen, "Interference measurements in the european $868 \mathrm{mhz}$ ism band with focus on lora and sigfox," Wireless Communications and Networking Conference (WCNC), pp. 1-6, May 2017.

[8] Y. D. Beyene, R. Jantti, O. Tirkkonen, K. Ruttik, S. Iraji, A. Larmo, $\mathrm{T}$. Tirronen, and J. Torsner, "Nb-iot technology overview and experience from cloud-ran implementation," IEEE Wireless Commun., vol. 24, no. 3, pp. 26-32, Jun. 2017.

[9] Y. Song, J. Lin, M. Tang, and S. Dong, "An internet of energy things based on wireless lpwan," Engineering, vol. 3, no. 4, pp. 460-466, Apr. 2017.

[10] D. Mishra, S. De, S. Jana, S. Basagni, K. Chowdhury, and W. Heinzelman, "Smart rf energy harvesting communications: Challenges and opportunities," IEEE Commun. Mag., vol. 53, no. 4, pp. 70-78, Apr. 2015.

[11] N. Harpawi et al., "Design energy harvesting device of uhf tv stations," in 8th Intl. Conf. Telecom. Systems Services and Applications (TSSA), pp. 1-6, Oct. 2014.

[12] S. Keyrouz, H. Visser, and A. Tijhuis, "Ambient rf energy harvesting from dtv stations," Antennas and Propagation Conference (LAPC), Loughborough, pp. 1-4, Nov. 2012.

[13] H. Nishimoto, Y. Kawahara, and T. Asami, "Prototype implementation of wireless sensor network using tv broadcast rf energy harvesting," in Adjunct Proc. of 12th ACM Int. conf. on Ubicomp'10., pp. 373-374, Sep. 2010.

[14] X. Lu, P. Wang, D. Niyato, D. I. Kim, and Z. Han, "Wireless networks with rf energy harvesting: A contemporary survey," IEEE Commun. Surveys Tutorials, vol. 17, no. 2, pp. 757-789, Apr. 2015. 
[15] Y. Zeng, Y.-C. Liang, A. T. Hoang, and R. Zhang, "A review on spectrum sensing for cognitive radio: challenges and solutions," EURASIP J. Adv. Signal Process., vol. 2010, no. 1, p. 381465, Jan. 2010.

[16] T. Yucek and H. Arslan, "A survey of spectrum sensing algorithms for cognitive radio applications," IEEE Commun. Surveys Tutorials, vol. 11, no. 1, pp. 116-130, Jan. 2009.

[17] Y.-C. Liang, Y. Zeng, E. C. Peh, and A. T. Hoang, "Sensing-throughput tradeoff for cognitive radio networks," IEEE Trans. Wireless Commun., vol. 7, no. 4, pp. 1326-1337, Apr. 2008.

[18] H. Sun, A. Nallanathan, C.-X. Wang, and Y. Chen, "Wideband spectrum sensing for cognitive radio networks: a survey," IEEE Wireless Commun., vol. 20, no. 2, pp. 74-81, Apr. 2013.

[19] Z. Tian and G. B. Giannakis, "Compressed sensing for wideband cognitive radios," in Proc. IEEE Int. Conf. Acoustics, Speech, and Signal Processing (ICASSP), Honolulu, HI, vol. 4, pp. IV-1357, Apr. 2007.

[20] Y. Gao, Z. Qin, Z. Feng, Q. Zhang, O. Holland, and M. Dohler, "Scalable and reliable iot enabled by dynamic spectrum management for $\mathrm{m} 2 \mathrm{~m}$ in lte-a," IEEE Internet Things J., vol. 3, no. 6, pp. 1135-1145, Dec. 2016.

[21] X. Zhang, Y. Ma, H. Qi, Y. Gao, Z. Xie, Z. Xie, M. Zhang, X. Wang, G. Wei, and Z. Li, "Distributed compressive sensing augmented wideband spectrum sharing for cognitive iot," IEEE Internet Things J., Aug. 2018.

[22] H. Qi, X. Zhang, and Y. Gao, "Channel energy statistics learning in compressive spectrum sensing," IEEE Trans. Wireless Commun., Dec. 2018.

[23] S. H. Kim and D. I. Kim, "Hybrid backscatter communication for wireless-powered heterogeneous networks," IEEE Trans. Wireless Commun., vol. 16, no. 10, pp. 6557-6570, Jul. 2017.

[24] W. Liu, K. Huang, X. Zhou, and S. Durrani, "Next generation backscatter communication: Theory and applications," arXiv preprint arXiv:1701.07588, Aug. 2017.

[25] K. Han and K. Huang, "Wirelessly powered backscatter communication networks: Modeling, coverage, and capacity," IEEE Trans. Wireless Commun., vol. 16, no. 4, pp. 2548-2561, Mar. 2017.

[26] V. Liu, A. Parks, V. Talla, S. Gollakota, D. Wetherall, and J. R. Smith, "Ambient backscatter: wireless communication out of thin air," in Proc. of ACM SIGCOMM, vol. 43, no. 4, pp. 39-50, Aug. 2013.

[27] D. Darsena, G. Gelli, and F. Verde, "Achievable information rates of ambient backscatter communications," arXiv preprint arXiv:1605.04805, May 2016.

[28] M. K. Simon and M.-S. Alouini, Digital communication over fading channels. John Wiley \& Sons, 2005, vol. 95.

[29] A. J. Coulson, A. G. Williamson, and R. G. Vaughan, "A statistical basis for lognormal shadowing effects in multipath fading channels," IEEE Transactions on Communications, vol. 46, no. 4, pp. 494-502, Apr. 1998. 